\title{
GONIOSCOPIC LASER SCLEROSTOMY VERSUS FILTRATION SURGERY IN A RABBIT MODEL
}

\author{
JEFFREY H. RABOWSKY, ANDRES J. DUKES and DAVID A. LEE \\ Los Angeles, California
}

\begin{abstract}
SUMMARY
In a prospective and randomised study, we compared the course of pulsed dye laser ab interno sclerostomy with the course of posterior lip sclerectomy in 25 rabbits. One eye of each rabbit was randomly selected to be treated with laser; the fellow eye underwent posterior lip sclerectomy. Intraocular pressure (IOP) determinations and slit lamp examinations were recorded pre-operatively, then every other day for 3 weeks on 21 of the rabbits. Histological examination was performed on the eyes of 3 randomly selected rabbits that were killed 72 hours post-operatively. Laser sclerostomy resulted in an average maximal drop in IOP of $9.5 \mathrm{mmHg}$ on post-operative day 1 , and posterior lip sclerectomy resulted in a similar drop of $10.5 \mathrm{mmHg}(p=0.43, t$-test). By life-table analysis, $88 \%$ of eyes returned to within $2 \mathrm{mmHg}$ of pre-operative IOP values within 7 days following either laser internal sclerostomy or posterior lip sclerectomy. There was no significant difference between laser internal sclerostomy and posterior lip sclerectomy eyes with respect to the number of days needed to return to within 2 mmHg of preoperative IOP values $(p=0.26$, sign test of the life-table analysis). Pulsed dye laser internal sclerostomy appears to be as effective as posterior lip sclerectomy in lowering IOP in the rabbit model.
\end{abstract}

Current methods of glaucoma filtration surgery depend on surgically creating a pathway through the sclera to allow subconjunctival flow of aqueous humour. These techniques have several disadvantages. Although primary conventional filtering surgery has a success rate approaching $95 \%$, many eyes with previous surgery or with inflammatory or neovascular glaucoma do not fare as well. ${ }^{1-4}$ Also, current filtration techniques subject the patient to the risks of local or general anaesthesia. ${ }^{5}$ Lastly, any

From: Jules Stein Eye Institute, Department of Ophthalmology, UCLA School of Medicine, Los Angeles, California, USA.

Correspondence to: David A. Lee, MD, Jules Stein Eye Institute, UCLA School of Medicine, 100 Stein Plaza Los Angeles, CA 90095-7004, USA. incisional procedure subjects the eye to the risks of endophthalmitis, vitreous loss and expulsive haemorrhage.,

Laser sclerostomy may have several potential advantages over conventional filtering surgery, including less manipulation of the conjunctiva, less risk of intraocular haemorrhage and less inflammatory response, all of which may lead to less postoperative scarring. Since failure of glaucoma filtering surgery is usually due to post-operative fibroblast proliferation, collagen deposition and subsequent bleb or sclerostomy scarring, laser sclerostomy may provide a means of enhancing the success of filtration procedures. $^{7-9}$

Several laser sclerostomy techniques have been reported. Using an ab externo technique, Hoskins and co-workers have performed sclerostomies with a THC:YAG laser. ${ }^{10,11}$ This technique has the theoretical disadvantage of requiring insertion of a probe subconjunctivally, thus disturbing the conjunctiva. Several $a b$ interno techniques using the argon, ${ }^{12}$ erbium-YAG ${ }^{13}$ neodymium-YAG ${ }^{14-17}$ and excimer lasers ${ }^{18}$ have also been described. All these techniques require the passage of a fibre-optic or sapphire probe across the anterior chamber, risking damage to the intraocular structures. March and colleagues first described the use of a contact lens to perform an $a b$ interno laser sclerostomy. ${ }^{19,20}$ Latina et al. ${ }^{21}$ have described an $a b$ interno approach to laser sclerostomy using a pulsed dye laser, iontophoresis of methylene blue stain, and a goniolens that minimally disturbs the conjunctiva. Clinical studies using $a b$ interno laser sclerostomy with the pulsed dye laser in patients with refractory or end-stage glaucoma have shown promising results. ${ }^{2,23} \mathrm{We}$ have found reactive black 5 stain to be more effective than methylene blue in the creation of sclerostomy with the pulsed dye laser. We used reactive black 5 stain for iontophoresis to compare the course of pulsed dye laser $a b$ interno sclerostomy with posterior lip sclerectomy. 


\section{MATERIALS AND METHODS}

This study was performed in accordance with the ARVO Resolution on the Use of Animals in Research. Thirty-nine pigmented rabbits weighing $1.5-3.0 \mathrm{~kg}$ were studied. Pre-operatively, intraocular pressure (IOP) was measured in both eyes under topical $0.5 \%$ proparacaine (Alcon, Humacao, PR) anaesthesia by pneumotonometry (Alcon, Applanation Pneumotonograph, Digilab, Cambridge, MA); the mean of three separate readings was recorded. External examination of the conjunctival injection, corneal clarity, anterior chamber reaction and lens clarity was performed with a Zeiss slit-lamp biomicroscope (Carl Zeiss, Germany) and graded on a scale of $0-3$ (Table I) by one observer masked to the procedure performed.

One eye of each rabbit was randomly selected to undergo laser sclerostomy; for comparison the fellow eye underwent posterior lip sclerectomy during the same treatment session. General anaesthesia was given using ketamine $50 \mathrm{mg} / \mathrm{kg}$ i.m. (Parke-Davis, Morris Plains, NJ) and xylazine $15 \mathrm{mg} / \mathrm{kg}$ i.m. (Mobay, Shawnee, KS). Iontophoresis of 3\% reactive black 5 stain (Aldrich Chemical, Milwaukee, WI) was used to enhance scleral absorption of the laser energy. Topical proparacaine $0.5 \%$ was applied via a cotton-tip applicator to the planned site of iontophoresis to enhance stain penetration. Fresh reactive black stain was reconstituted from powder to $3 \%$ solution with double-distilled water for each treatment session. The stain was iontophoresed at the posterior surgical limbus for 5 minutes at $500 \mathrm{~mA}$ using a constant current source. The iontophoresis

Table I. Scales used for slit-lamp parameters

\begin{tabular}{ll}
\hline Parameter & Description \\
\hline Conjunctival injection & No injection \\
0 & Trace injection \\
1 & Moderate injection \\
2 & Severe injection with chemosis \\
3 & \\
Corneal clarity & No opacity \\
0 & Opacity involving $<1 / 3$ of the cornea, \\
1 & and not obscuring iris detail \\
2 & Opacity involving $1 / 3-2 / 3$ of the cornea \\
& and not obscuring iris detail, or $<1 / 3$ \\
& obscuring iris detail \\
3 & Opacity involving $>2 / 3$ of the cornea \\
& and not obscuring iris detail, or \\
3 & involving $>1 / 3$ obscuring iris detail \\
3 & No flare or cells \\
3 & Mild flare, no cells \\
1 & Moderate flare with cells \\
Lens clarity & Severe flare with cells and fibrin \\
&
\end{tabular}

probe consisted of a plastic tube with a tubular Porex (Porex Technologies, GA) sponge inside. The tip of the probe was tapered to a final dimension of $0.5 \times 0.5 \mathrm{~mm}$. All exposed areas of the probe tip except the distal $0.5 \mathrm{~mm}$ were coated with $100 \%$ silicone rubber (RTV Sealant, Dow Corning, Midland, MI). Electrical contact with the sponge was via a silver-silver chloride wire. The positive electrode of the current source served as a ground on the rabbit's ipsilateral ear, and the negative electrode was placed on the iontophoresis probe. The probe was kept moist between each use by storing it in reactive black 5 stain solution. After iontophoresis, excess stain was removed from the eye surface by blotting the stained area and irrigating the eye with sterile saline. Prior to the laser application, a conjunctival bleb was raised with $0.2 \mathrm{ml}$ sterile saline using a 30-gauge needle in order to prevent conjunctival perforation by the laser energy. Care was taken to keep the site of needle entry away from the intended sclerotomy site. Using a cotton-tip applicator, the bleb was then massaged as far anteriorly as possible to lift the conjunctiva off the stained scleral surface. Pilot studies showed that this procedure did not increase conjunctival scarring.

A flashlamp-pumped pulsed dye laser was provided by Candela Laser (Wayland, MA). The laser output was coupled to a slit-lamp delivery system by a fibre-optic cable that provided a $200-\mu \mathrm{m}$ spot size. A wavelength of $590 \mathrm{~nm}$, duration of $8 \mu$ s and energy of approximately $150 \mathrm{~mJ}$ were used. Prior to treatment of each rabbit, the laser output was measured on an energy meter (Model 365 power and energy meter, Scientech, Boulder, $\mathrm{CO}$ ).

A modified Zeiss goniolens (supplied by Candela Laser, Wayland, MA) was used to deliver the laser energy. The Zeiss lens was modified by removing the reflective mirror coating, then the polished glass reflecting surfaces were coated with aluminium foil and epoxy resin for protection. Thus, the laser energy was directed entirely by internal reflection and the posterior angle structures were visualised by

Table II. Complications

\begin{tabular}{lc}
\hline Types of complications & Number \\
\hline Leading to exclusion & \\
Anaesthetic-related death & 1 \\
Laser sclerostomy eyes: & 1 \\
$\quad$ Inadequant dye iontophoresis & 5 \\
$\quad$ Failure of chamber to shallow during lasering & 4 \\
$\quad$ Seidel-positive & \\
Posterior lip sclerectomy eyes: & 2 \\
$\quad$ Vitreous loss & 1 \\
$\quad$ Seidel-positive & \\
Not leading to exclusion & \\
Laser sclerostomy eyes: & 1 \\
$\quad$ Hyphaema & 5 \\
Subconjunctival haemorrhage & \\
Posterior lip sclerectomy eyes: & 6 \\
\hline Hyphaema & \\
\hline
\end{tabular}




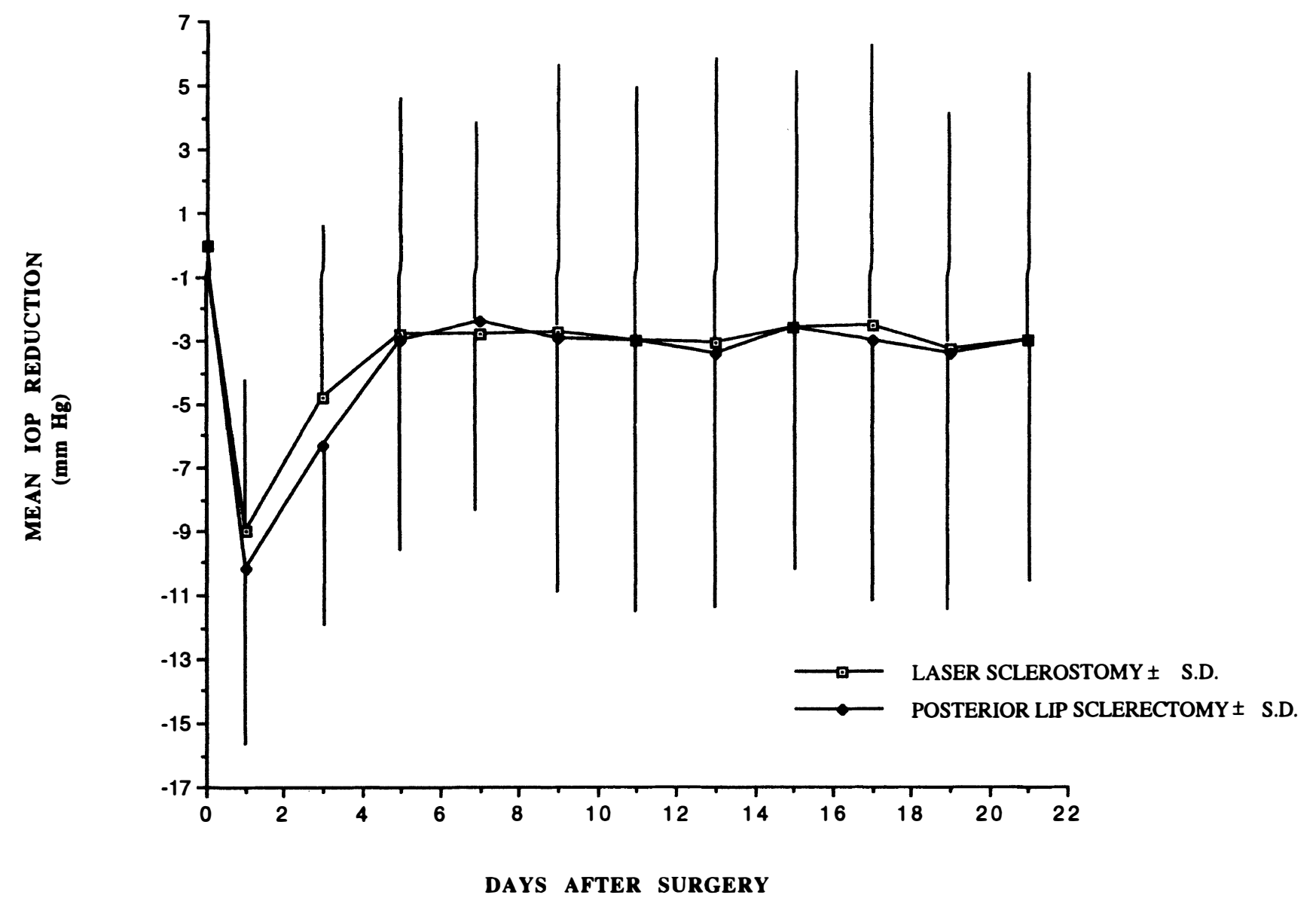

Fig. 1. Mean post-operative change in IOP from baseline for eyes treated with pulsed dye laser ab interno sclerostomy versus eyes treated with posterior lip sclerectomy. Error bars indicate $1 S D$.

compression gonioscopy during the treatment. The laser was aimed via a helium-neon beam focused on the most posterior aspect of the dye spot. This area was always anterior to the top of the trabecular meshwork (insertion of the iris pillars to the cornea in the rabbit eye). The laser was repeatedly fired until the anterior chamber shallowed and bleb formation was visualised. Care was taken to ensure that all the spots overlapped. If the anterior chamber failed to shallow, the procedure was considered a failure. No peripheral iridectomy was performed and there was no immediate obstruction of the sclerostomy by iris in any of the eyes tested. ${ }^{23}$ Postoperatively, the external region of the sclerostomy was examined under an operating microscope and a Seidel test was performed using a fluoresceinimpregnated strip of paper (Ayerst, New York, NY). Tobramycin ointment (Alcon Laboratories, Forth Worth, TX) then was applied to the surface of the eye.

Thereafter, posterior lip sclerectomy was performed on the fellow eye. A lid speculum was applied and a superotemporal, limbal-based conjunctival flap was made with Wescott scissors. The conjunctiva was bluntly dissected forwards using a cotton-tip applicator. Excessive Tenon's tissue was removed. A groove was made with a 64 Beaver blade (Rudolph Beaver, Waltham, MA) just posterior to the limbus and a short scleral tunnel was made into clear cornea. The anterior chamber was then entered at the sclerectomy site with a 15-degree Alcon superblade (Alcon Surgical, Fort Worth, TX). A $2 \times 2 \mathrm{~mm}$ block of limbal tissue was excised by taking two separate bites with a Kelly-Descemets punch to create a full-thickness sclerectomy. Cautery was employed as necessary to achieve haemostasis. A peripheral iridectomy was performed using a 0.12 forceps and Vannas scissors. The conjunctiva was closed with a running, intermittently locked 8-0 vicryl suture. A Seidel test then was performed over the surgical site, and tobramycin ointment applied to the surface of the eye.

Rabbits with a wound leak, positive Seidel test, eight-ball hyphaema or failure of the anterior chamber to shallow during the laser sclerostomy were killed at the time of surgery and not included in the study. Post-operative follow-up included slitlamp examination and IOP measurements every 


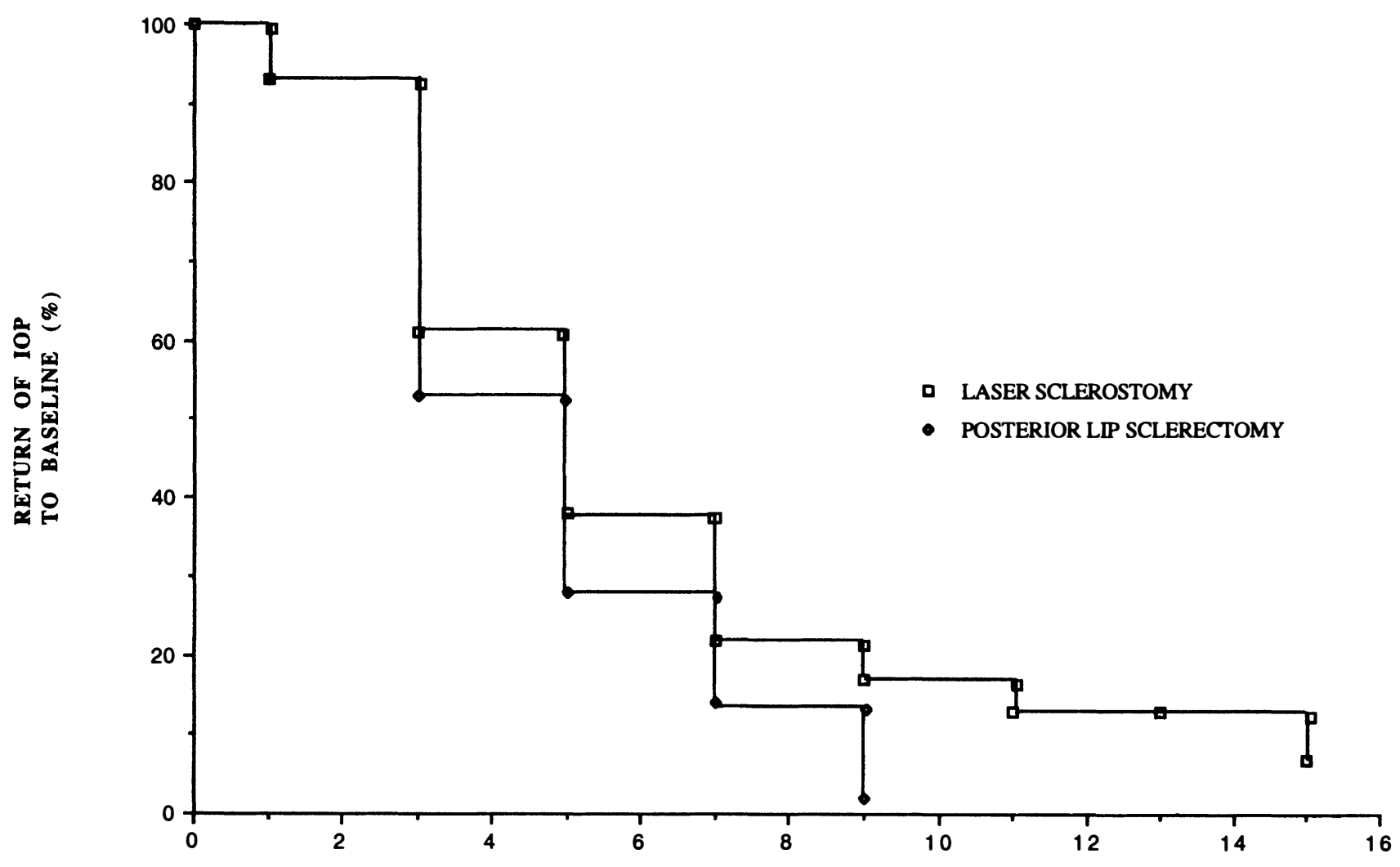

DAYS AFTER SURGERY

Fig. 2. Life-table analysis of the time required for IOP to return to within $2 \mathrm{mmHg}$ of the mean pre-operative value for eyes treated with pulsed dye laser ab interno sclerostomy versus eyes treated with posterior lip sclerectomy.

other day for 3 weeks, starting on the first postoperative day. In addition, the presence or absence of a bleb was noted during each examination. The examinations were performed by one observer who was masked to the procedure performed in each eye.

Twenty-one animals were killed with an overdose of sodium pentobarbital $75 \mathrm{mg} / \mathrm{kg}$ i.v. 3 weeks after surgery. Three rabbits were selected using an algorithm based on a random number table, and killed 72 hours after surgery for histopathological examination. The eyes from these animals were enucleated and fixed in $10 \%$ neutral-buffered formalin for 48 hours. The eyes were then sectioned in the sagittal plane into two calottes, and embedded in paraffin. Step sections $5 \mu \mathrm{m}$ thick then were obtained and stained with haematoxylin-eosin and Masson's trichrome.

Statistical analysis was performed to compare, for each rabbit, the eye that underwent laser sclerostomy with the fellow eye that underwent posterior lip sclerectomy. Variables examined included IOP; time to return of IOP to within 1,2 or $3 \mathrm{mmHg}$ of the baseline pressure; time to bleb failure; and time to return to baseline for conjunctival injection, anterior chamber reaction, corneal clouding and cataract formation. The variables were compared using the paired $t$-test for all the rabbits followed for 3 weeks $(n=21)$. Life-table analysis using McNemar's statistic for paired data was used as appropriate to compare all rabbits included in the study $(n=25){ }^{24,25}$ A probability of less than 0.05 was considered to be statistically significant for all analyses.

\section{RESULTS}

Of a total of 39 rabbits operated on, 14 were excluded from the study. One rabbit that died from an unknown cause on the fifth day of follow-up was included in the data analysis. Complications not leading to exclusion in the lasered eyes included 1 eye that developed a 5\% hyphaema and 5 eyes that developed small subconjunctival haemorrhages. Of the surgical eyes, 6 developed 5-50\% hyphaemas (Table II).

Average measured energy per laser shot $( \pm S D)$ was $156 \pm 5.2$ (range 148-167) $\mathrm{mJ}$. An average of $225 \pm 111$ (range 25-486) shots were required to obtain the laser clinical success endpoint. The average total energy used per lasered eye was 


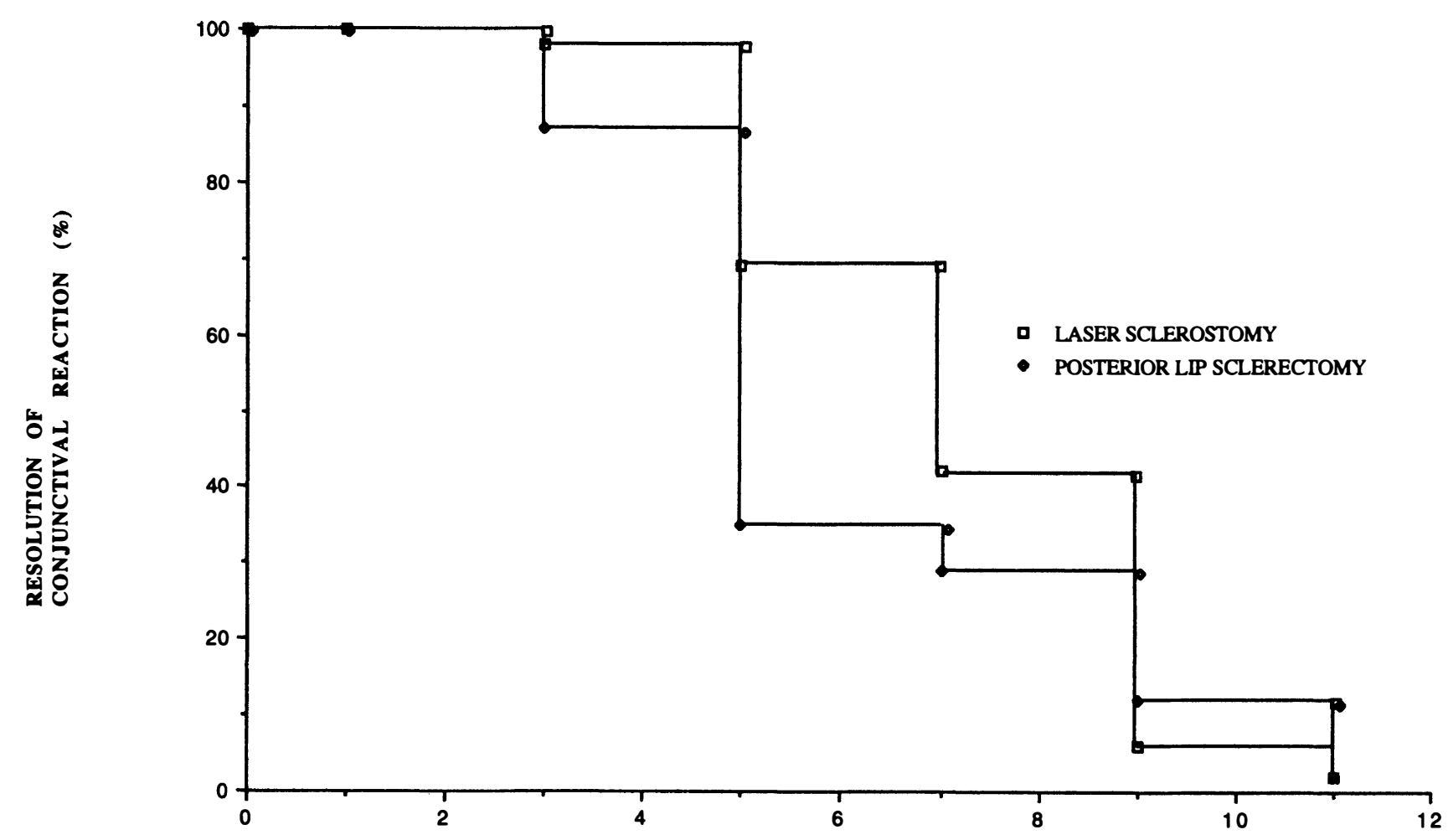

DAYS AFTER SURGERY

Fig. 3. Life-table analysis of the time required for conjunctival reaction to return to baseline values for eyes treated with pulsed dye laser ab interno sclerostomy versus eyes treated with posterior lip sclerectomy.

$35.0 \pm 17.3$ (range 3.9-76.8) J. It required approximately 10 minutes to complete the laser treatment in each eye.

Pre-operatively, there was no statistically significant difference in IOP between laser sclerostomy and posterior lip sclerectomy eyes ( $t$-test, $p=1.00)$. There was no significant difference between laser sclerostomy and posterior lip sclerectomy with respect to either the extent or the duration of the drop in IOP on any post-operative day (Fig. 1). Power calculations yielded a $90 \%$ power in detecting a $3 \mathrm{mmHg}$ difference in IOP with the sample size used in this study. The maximum drop in IOP occurred on the first day. This drop was $9.5 \mathrm{mmHg}$ for lasered eyes. By life-table analysis, $88 \%$ of both laser sclerostomy and posterior lip sclerectomy eyes returned to within $2 \mathrm{mmHg}$ of pre-operative IOP within 7 days (Fig. 2).

As revealed by slit-lamp examination, conjunctival reaction returned to baseline in significantly fewer days in the laser sclerostomy eyes than in the posterior lip sclerectomy eyes $(p=0.02$, sign test of life-table analysis; Fig. 3). This relationship occurred for the anterior chamber reaction as well $(p=0.002$; Fig. 4). There was no significant difference between laser sclerostomy and posterior lip sclerectomy eyes with regard to corneal clarity, lens clarity or bleb survival. The mean time ( $\pm S D$ ) for the stain to disappear was $6.4 \pm 3.1$ days.

Histological examination performed on the eyes of 3 rabbits killed 72 hours after surgery showed a

Table III. Laser sclerostomy and posterior lip sclerectomy eyes (\%) with slit-lamp parameter score ${ }^{\mathrm{a}}>0(n=23)$

\begin{tabular}{|c|c|c|c|c|c|c|}
\hline \multirow[b]{2}{*}{ Day } & \multicolumn{2}{|c|}{ Conjunctival injection } & \multicolumn{2}{|c|}{ Corneal clarity } & \multicolumn{2}{|c|}{ Anterior chamber inflammation } \\
\hline & Laser sclerostomy & Posterior lip sclerectomy & Laser sclerostomy & Posterior lip sclerectomy & Laser sclerostomy & Posterior lip sclerectomy \\
\hline 1 & 100 & 100 & 33.3 & 14.3 & 80.9 & 90.5 \\
\hline 3 & 76.2 & 95.2 & 28.6 & 9.5 & 33.3 & 76.2 \\
\hline 5 & 28.6 & 23.8 & 19.0 & 9.5 & 19.5 & 42.9 \\
\hline 7 & 23.8 & 19.0 & 9.5 & 0 & 0 & 0 \\
\hline 9 & 9.5 & 4.8 & 9.5 & 0 & 0 & 0 \\
\hline 11 & 0 & 0 & 9.5 & 0 & 0 & 0 \\
\hline 13 & 0 & 0 & 9.5 & 0 & 0 & 0 \\
\hline
\end{tabular}

${ }^{\text {a See Table I. }}$ 


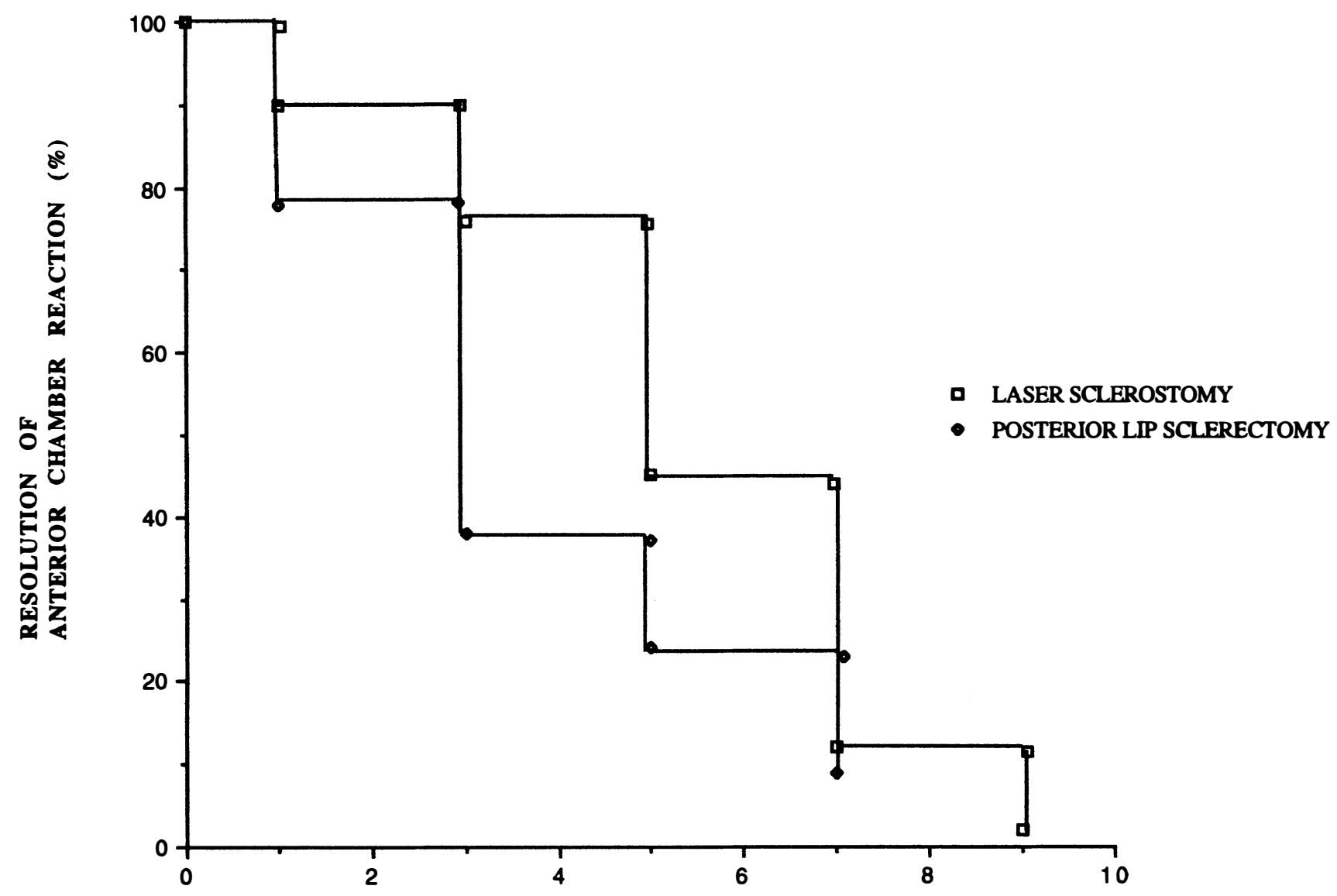

DAYS AFTER SURGERY

Fig. 4. Life-table analysis of the time required for anterior chamber reaction to resolve for eyes treated with pulsed dye laser $\mathrm{ab}$ interno sclerostomy versus eyes treated with posterior lip sclerectomy.

partial sclerostomy through the limbus of those eyes treated with the laser (Fig. 5). A patent sclerectomy was demonstrated in all 3 eyes that received posterior lip sclerectomies (Fig. 6). No inflammation in the ciliary body or cyclodialysis clefts was observed.

\section{DISCUSSION}

We found pulsed dye laser internal sclerostomy and conventional filtering surgery to be equally effective in lowering IOP in the rabbit model. In addition, we found that there was significantly less conjunctival and anterior chamber reaction in the lasered eyes. Theoretically, this lower inflammatory response could lead to less aggressive wound healing and scarring, resulting in improved filtration success.

In eyes with extensive conjunctival scarring, laser sclerostomy offers an alternative to difficult and often risky conjunctival dissection. With a potentially lower complication rate and less serious complications, it may be safe to repeat laser sclerostomy several times anywhere around the limbus to reduce IOP in a graded manner. Lastly, laser sclerostomy may allow filtering surgery to be performed entirely on an outpatient basis under topical anaesthesia.

Laser sclerostomy was technically easier and faster to perform than posterior lip sclerectomy. The two procedures posed different sets of complications. Inaccurate stain placement may lead to inability to perform the laser sclerostomy. This problem can be solved by repeating the iontophoresis. Failure of the laser to penetrate the sclera occurred in 5 of 38 attempted treatments. The most serious complication encountered with laser sclerostomy was perforation of the cornea at or anterior to the conjunctival insertion into the cornea, leading to wound leak. Rabbits are especially predisposed to this complication because the angle width is relatively narrow and the conjunctival insertion is posterior to that in humans. ${ }^{26}$ In order to decrease the risk of conjunctival perforation, the sclerostomy was always placed in the most posterior aspect of the stain spot. The only other complications with the laser sclerostomy were a greater incidence of subconjunctival haemorrhage than that observed in posterior lip sclerectomy eyes, and small, localised areas of corneal haze, possibly 


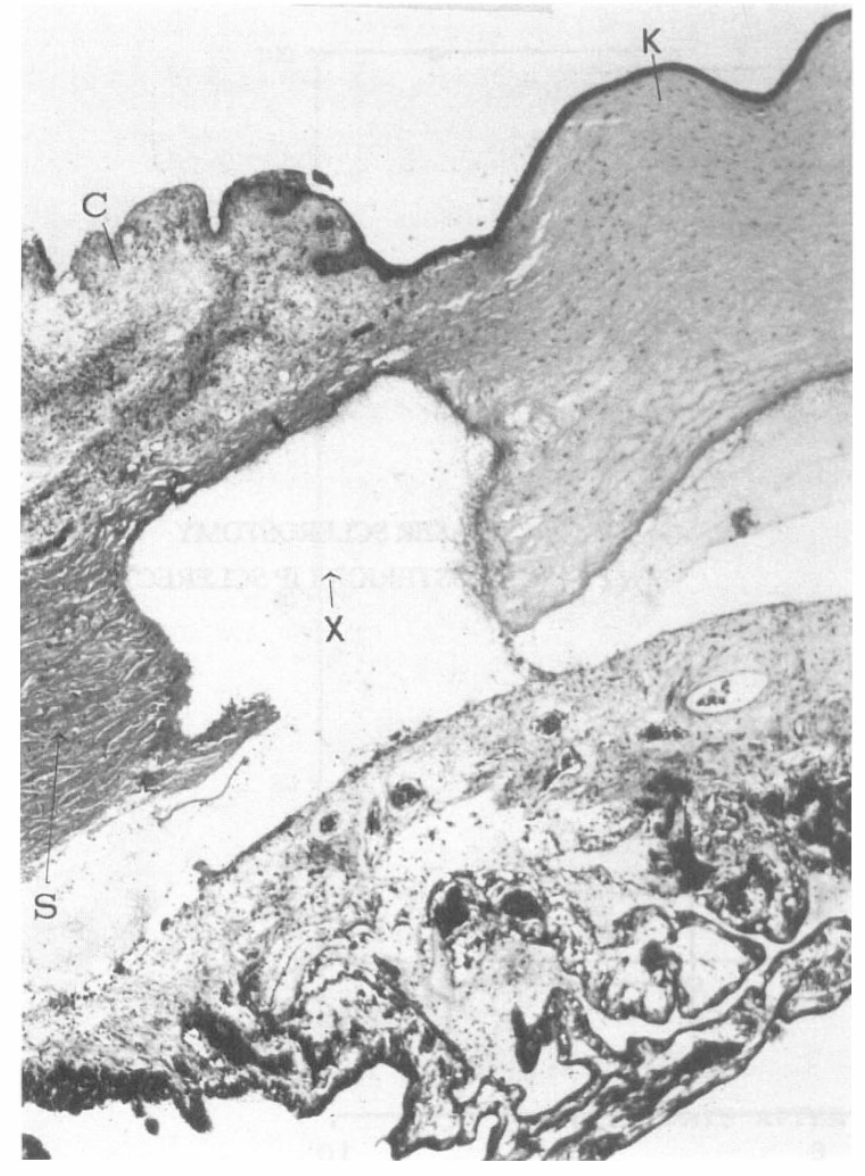

Fig. 5. Photomicrograph of pulsed dye laser ab interno sclerostomy in the rabbit eye. Partial-thickness sclerostomy is present. $K$, cornea; $X$, fistula; $S$, sclera; $C$, conjunctiva (Masson's trichrome, original magnification $\times 25$ ).

related to endothelial damage, around the sclerostomy sites. As noted above, the human eye has a deeper anterior chamber with a wider approach to the angle structures than does the rabbit eye. In addition, the conjunctiva inserts more anteriorly on the cornea. ${ }^{26,27}$ Thus, it should be easier to place the sclerostomy and avoid conjunctival perforation in humans than in rabbits.

The total average energy required to perform laser sclerostomy in our study was much higher than that reported by Latina ${ }^{21}$ and others. ${ }^{22,23}$ This disparity may have been due to the more posterior placement of the sclerostomy in our study. A major factor that was found to influence energy required for perforation was the anterior-posterior relationship of the sclerostomy to the iris insertion. More anteriorly placed sclerostomies that resulted in perforation of the conjunctival insertion required much less energy for perforation (average total energy, $4.41 \mathrm{~J}$ ) than did posteriorly placed sclerostomies (average total energy, $35.0 \mathrm{~J}$ ). The posterior region of the stain spot could have had a lower concentration of stain, leading to less efficient energy absorption. In addition, as the laser beam is aimed more posteriorly,

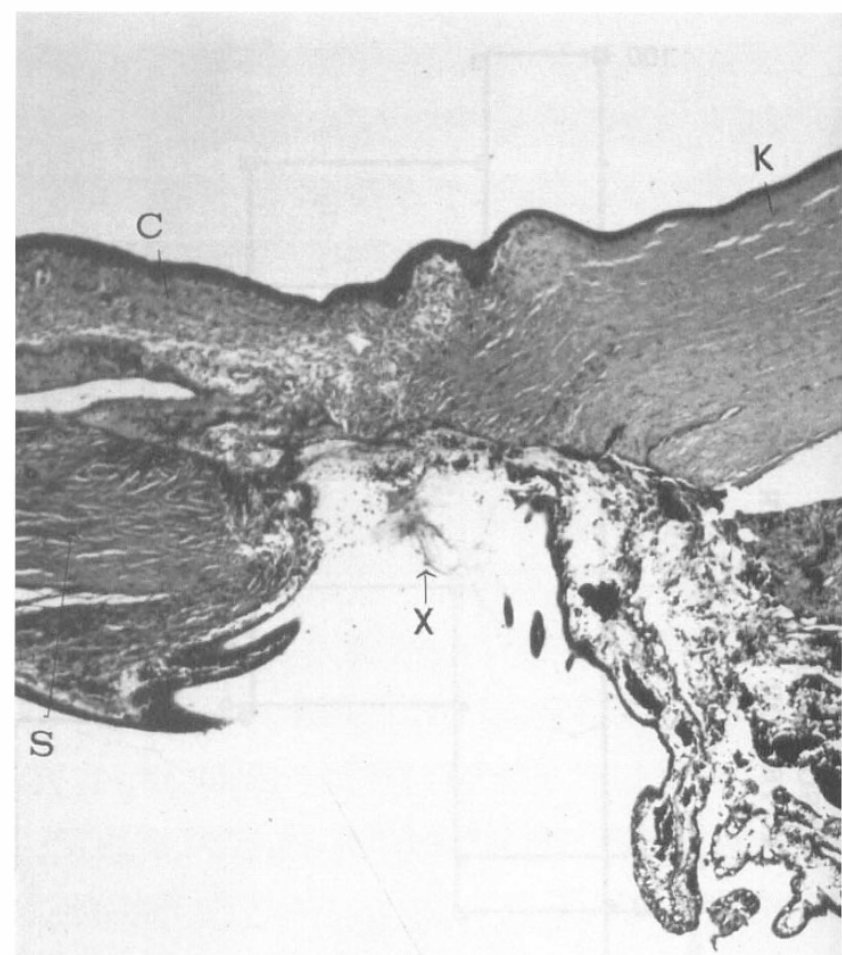

Fig. 6. Photomicrograph of posterior lip sclerectomy in the rabbit eye. Full-thickness sclerostomy is present. $K$, cornea; $X$, fistula; $S$, sclera; $C$, conjunctiva (Masson's trichrome, original magnification $\times 25$ ).

it forms a more oblique angle of incidence to the sclera, possibly leading to less efficient tissue ablation and a longer sclerostomy tract.

Reactive black 5 was employed in this study rather than methylene blue. In preliminary trials using the laser technique employed in this study, we had found methylene blue to bleach after 10-20 shots, resulting in a poor rate of scleral perforation. No stain bleaching was observed during the rabbit treatments in this study.

There may be several mechanisms of action for the IOP-lowering effect of pulsed dye laser internal sclerostomy. Filtration may have played a role in many eyes. In the lasered eyes, the IOP survival curve closely matched the survival curve for the presence of filtering blebs. Our pulsed dye laser sclerostomies were approximately $300 \mu \mathrm{m}$ in width, compared with the posterior lip sclerectomies which were $2000 \mu \mathrm{m}$ wide. The laser sclerostomies might be expected to close sooner than the posterior lip sclerectomies. ${ }^{27}$ Increased outflow could have occurred transsclerally or from uveoscleral outflow as a result of scleral damage from a partial sclerostomy.

The decreased IOP after laser sclerostomy may be due to decreased aqueous production secondary to 
inflammation. Cyclodialysis clefts may have been created, either because treatment was too posterior in the anterior chamber angle or as a result of acoustic effects of the laser. However, histopathological examination did not show ciliary body inflammation or cyclodialysis clefts.

Pulsed dye laser internal sclerostomy appears to be as effective in IOP reduction as posterior lip sclerectomy in the rabbit model. Additional studies to control the post-operative course of laser sclerostomy in rabbits using modulators of the wound healing process may be valuable. In the future, prospective, randomised human studies comparing laser sclerostomy with conventional filtration surgery may be beneficial.

This work was supported by NIH grants EY 07701, EY 00331, EY 08019, a UCLA Academic Senate grant, and the Lucille Ellis Simon Glaucoma Research Fund. J.H.R. is a Head Ophthalmic Foundation Fellow and Lee and Mae Sherman Fellow. The authors gratefully acknowledge Saeedeh Shapourifar-Tehrani, MPH, for her help in preparing the histopathology specimens for this study and Sarah B. Forsythe, MS, for her assistance with statistical analysis. The equipment and assistance provided by Candela Laser Corporation are also appreciated.

Each author states that he has no proprietary interest in the development or marketing of this or a competing instrument.

Key words: Filtering surgery, Laser, Sclerostomy, Iontophoresis, Glaucoma.

\section{REFERENCES}

1. Shields MB. Anatomic principles of glaucoma surgery. In: Kist $\mathrm{K}$, editor. Textbook of glaucoma. Baltimore: Williams and Wilkins, 1987:458-87.

2. Heuer DK, Gressel MG, Parrish RK, et al. Trabeculectomy in aphakic eyes. Ophthalmology 1984;91: 1045-51.

3. Jampel HD, Jabs DA, Quigley HA. Trabeculectomy with 5-fluorouracil for adult inflammatory glaucoma. Am J Ophthalmol 1990;109:168-73.

4. Allen RC, Bellows AR, Hutchinson BT, Murphy SD. Filtration surgery in the treatment of neovascular glaucoma. Ophthalmology 1982;89:1181-6.

5. Krupin T, Waltman SR. Complications in ophthalmic surgery. Philadelphia: JB Lippincott, 1984:1-22.

6. Katz LJ, Cantor LB, Spaeth GL. Complications of surgery in glaucoma: early and late bacterial endophthalmitis following glaucoma filtering surgery. Ophthalmology 1985;92:959-63.

7. Maumenee AE. External filtering operations for glaucoma: the mechanism of function and failure. Trans Am Ophthalmol Soc 1960;58:319-28.

8. Addicks EA, Quigley HA, Green R, Robin AL. Histologic characteristics of filtering blebs in glaucomatous eyes. Arch Ophthalmol 1983;101:795-8.
9. Tahery M, Lee DA. Review: pharmacologic control of wound healing in glaucoma filtration surgery. J Ocular Pharmacol 1989;5:155-79.

10. Hoskins HD Jr, Iwach AG, Drake MV, Schuster BL, Vassiliadis A, Crawford JB, Hennings DR. Subconjunctival THC:YAG laser limbal sclerostomy $a b$ externo in the rabbit. Ophthalmic Surg 1990;21:589-92.

11. Hoskins HD Jr, Iwach AG, Vassiliadis A, Drake M, Hennings DR. Subconjunctival THC:YAG laser thermal sclerostomy. Ophthalmology 1991;98:1394-400.

12. Jaffe GJ, Williams GA, Mieler WF, Radius RL. $A b$ interno sclerostomy with a high-powered argon endolaser. Am J Ophthalmol 1988;106:391-6.

13. Berlin MS, Rajacich G, Duffy M, et al. Excimer laser photoablation in glaucoma filtering surgery. Am J Ophthalmol 1987;103:713-4.

14. Gherezghiher T, March WF, Koss MC, Nordquist RE. Neodymium-YAG laser sclerostomy in primates. Arch Ophthalmol 1985;103:1543-5.

15. March WF, Gherezghiher T, Koss MC, Nordquist RE. Histologic study of a neodymium:YAG laser sclerostomy. Arch Ophthalmol 1985;103:860-3.

16. Javitt JC, O'Connor SS, Wilson RP, Federman JL. Laser sclerostomy ab interno using a continuous wave neodymium:YAG laser. Ophthalmic Surg 1989; 20:552-6.

17. Higginbotham EJ, Kao G, Peyman GA. Internal sclerostomy with a Nd:YAG contact laser vs thermal sclerostomy in rabbits. Ophthalmology 1988;95:385-90.

18. Margolis TI, Farnath DA, Puliafito CA. Midinfrared laser sclerostomy. Invest Ophthalmol Vis Sci 1988;29(Suppl):366.

19. March WF, Gherezghiher T, Koss MC, Nordquist RE. Experimental YAG laser sclerostomy. Arch Ophthalmol 1984;102:1834-6.

20. March WF, La Fuente H, Rol P. Improved goniolens for YAG sclerostomy. Ophthalmic Surg 1987;18:513.

21. Latina MA, Dobrogowski M, March WF, Birngruber R. Laser sclerostomy by pulsed-dye laser and goniolens. Arch Ophthalmol 1990;108:1745-50.

22. Ruben S, Migdal C, DeVivero C. Ab interno pulsed dye laser sclerostomy for the treatment of glaucoma: preliminary results of a new technique. Eye 1993;7: 436-9.

23. Melamed S, Solomon A, Neumann D, Hirsh A, Blumenthal M, Belkin M. Internal sclerostomy using laser ablation of dyed sclera in glaucoma patients: a pilot study. Br J Ophthalmol 1993;77:139-44.

24. Holt JD, Prentice RL. Survival analysis in twin studies and matched pair experiments. Biometrika 1974; 61:17-30.

25. Seigel D, Podgor M. A sign test for significance of differences in survivorship curves from paired truncated data. Controlled Clin Trials 1982;3:69-71.

26. Troncoso MU, Castroviejo R. Microanatomy of the eye with the slit-lamp microscope. 1. Comparative anatomy of the angle of the anterior chamber in living and sectioned eyes of Mammalia. Am J Ophthalmol 1942;19:371-84.

27. Miller MH, Joseph NH, Ennis KW, et al. An animal model of filtration surgery. Trans Ophthalmol Soc UK 1985;104:893-7. 